\title{
EFFECTS OF DIFFRACTION AND SCREEN SIZE ON CTR BASED BUNCH LENGTH MEASUREMENTS
}

\begin{abstract}
M. Castellano, A. Cianchi, V. A. Verzilov
Abstract

Coherent transition radiation (CTR) is now well established as one of basic tools to measure electron bunches on a subpicosecond scale. However, a series of experiments has demonstrated that suppression of CTR spectra at low frequencies, occurring in practice, leads to a great uncertainty in the bunch length determination. In addition to known sources of suppression, it was recently found $[1,2]$ that the size of a screen used to produce transition radiation (TR) can be a factor strongly affecting the spectrum. In this paper we calculate TR spectra emitted by a relativistic electron from a finite-size metallic screen and influenced by diffraction on apertures in environment and collecting optics.
\end{abstract}

\section{INTRODUCTION}

Operating with subpicosecond bunches is crucial to the new generation of $e^{+} e^{-}$colliders and FELs for reaching their final goals, which are respectively high luminosity and high peak current. To obtain this result, much depends on the ability to monitor bunch dimensions on such a small scale.

Coherent transition radiation (CTR) is now intensively used for ultra-short bunch length measurements due to its simplicity of implementation and small perturbations produced to the beam.

In this technique the bunch longitudinal dimension can be extracted from the measured CTR spectrum if the incoherent TR spectrum is precisely known [3]. In practice, however, there is a number of experimental factors, such as the limited bandwidth of the detector and diffraction effects due to finite apertures in the radiation transport channel, which cause hardly evaluated losts of the low frequency part of measured spectra, thus leading to a considerable uncertainty in the bunch length and shape determination [4].

Furthermore, the proper role of the size of the target in modifying the power spectrum of incoherent TR was shown $[1,2]$ recently. It was found that the TR spectrum from a finite target is a complex function of the beam energy, target extensions, frequency and angle of emission, i.e, very different from the flat spectrum given by the Frank formula, that has been used so far. The effect occurs when the parameter $\gamma \lambda$, where $\gamma$ is the relativistic factor of the beam and $\lambda$ is the radiation wavelength, exceeds the transverse dimensions of the target.

\footnotetext{
*Email: verzilov@lnf.infn.it
}

\section{INFN-LNF, G. Orlandi INFN-Roma2 \\ 2 EFFECTS OF DIFFRACTION AND TARGET SIZE ON TR SPECTRA}

In the pioneering works [1] the treatment of the problem was performed for a thin layer of matter and TR emitted in the forward direction. As a consequence, the resulting picture of the effect includes interference between TR, the particle field and diffraction radiation and, therefore, is rather complex.

In the present analysis, we rely on the Kirchoff diffraction theory to describe the propagation of the field generated by a charged particle on the boundary between the vacuum and a perfect conducting material [5].TR is considered to emerge in the backward directions with respect to the momentum of the particle crossing the boundary at normal incidence. Three different schemes of measurement, which are simplified models of those typically encountered in practice, are investigated.

\subsection{Spectrum of TR filtered by a finite aperture}

The first scheme considered is characterized by the presence of a circular diaphragm between the emitting screen and the detector. For ease of calculation we assume a cylindrically symmetric geometry. A circular screen with a radius $r$ made of a perfect metal is placed at a distance $a$ from the diaphragm, while $b$ is the distance between the diaphragm and the detector having a diameter $2 d$ ( Fig. 1a). The incident particle with charge $q$ and velocity $v$ hits the screen at the center. Emerging TR propagates in the $z$ direction. Let us introduce three different sets of coordinates $\left(x_{s}, y_{s}\right),(\xi, \eta)$ and $(x, y)$ for the screen, diaphragm and detector planes, respectively.

In cylindrical coordinates we have:

$$
\left\{\begin{array}{l}
x_{s} \\
y_{s}
\end{array}\right\}=\rho_{s}\left\{\begin{array}{c}
\cos \varphi \\
\sin \varphi
\end{array}\right\},\left\{\begin{array}{l}
x \\
y
\end{array}\right\}=\rho\left\{\begin{array}{c}
\cos \chi \\
\sin \chi
\end{array}\right\}
$$

Then the TR field components at an arbitrary point $\mathrm{P}(\mathrm{x}, \mathrm{y})$ in the detector plane in the first order Fresnel approximation of the diffraction theory, and neglecting phase constants, are found to be

$$
\begin{array}{r}
E_{x, y}(P, \omega)=\frac{q}{2 \pi^{2} v} \frac{k^{2}}{a b} \int \mathrm{d} \rho_{s} \rho_{s} \int \mathrm{d} k_{\perp} \frac{k_{\perp}^{2} J_{1}\left(k_{\perp} \rho_{s}\right)}{k_{\perp}^{2}+\alpha^{2}} \\
\times \mathrm{e}^{\mathrm{i}(k / 2 a m) \rho_{s}^{2}} \int \mathrm{d} \varphi\left\{\begin{array}{l}
\cos \varphi \\
\sin \varphi
\end{array}\right\} \mathrm{e}^{-\mathrm{i}(k / a m) \rho \rho_{s} \cos (\varphi-\chi)} \mathcal{L}(p),
\end{array}
$$

where $k_{\perp}$ is the projection on the xy plane of the the photon wave vector $k=\omega / c, \alpha=\omega / v \gamma, m=1+b / a, J_{1}$ is the Bessel function of the first kind and

$$
\mathcal{L}(p)=2 \pi \mathrm{e}^{\mathrm{i}(k / 2 b m) p^{2}} \int_{0}^{D} \mathrm{~d} \zeta \zeta \mathrm{e}^{\mathrm{i}(k m / 2 b) \zeta^{2}} J_{0}(k \zeta p / b),
$$



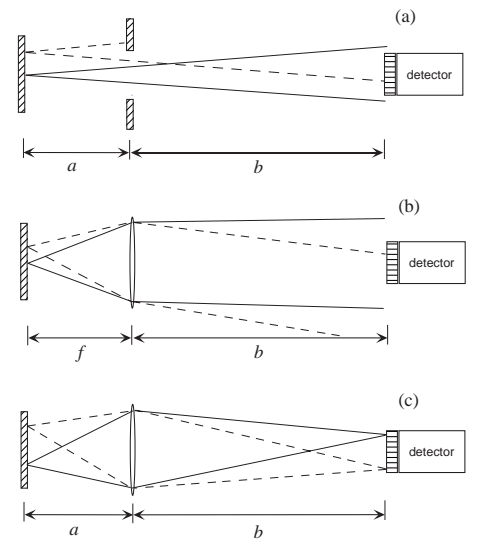

Figure 1: Three schemes of measurements under consideration.

$$
p=\sqrt{\rho^{2}+\left(b^{2} / a^{2}\right) \rho_{s}^{2}+2(b / a) \rho \rho_{s} \cos (\varphi-\chi)},
$$

As follows from Eq. (2), the field in $\mathrm{P}$ is built up by a coherent summation of the waves emitted by all points of the source and so it depends on both the shape and the size of the screen. The function $\mathcal{L}$, mainly determined by the integral over the diaphragm surface, gives a contribution from the standard diffraction by the aperture. The above integral is well known (see, e.g., [6]) and is expressed in terms of the Lommel functions.

Thus, Eq. (2) includes effects given by both the size of the screen and the diffraction produced by the diaphragm. The latter is well known to produce, basically, a low frequency spectrum cutoff, almost entirely defined by the diaphragm aperture and angular acceptance. Hence, from this point on, we found it reasonable to focus our study on that of the screen size. To this end we formally let $D$ tend to $\infty$.

In terms of the theory of radiation, the phase factor quadratic in $\rho_{s}$ in Eq. (2) specifies first order corrections to the so-called wave zone (or radiation zone) approximation due to the extension of the source and the sphericity of wave fronts at the point $\mathrm{P}$.

For a finite-size screen these corrections are noticeable if

$$
\frac{r^{2}}{a m} \geq \lambda \geq \frac{a m}{\gamma^{2}}
$$

and their effect is, in last instance, to reduce the "effective" size of the screen.

It should be noted that these corrections are relevant even for an infinite screen causing it to act like a finitesize one with an "effective" dimension depending on the wavelength and the distance to the observation point, if TR is observed at distances

$$
\lambda \gamma^{2} \geq z
$$

In the far-infrared region, that represents our main interest, the wave-zone condition can be well fulfilled by adjusting the distance between the target and detector, thus allowing to approximate the aforementioned phase factor by 1 .

As a consequence of simplifications made, the total spectrum of TR from the finite-size screen, integrated over the detector aperture, in the wave zone is found from Eq. (2) upon a change of variable $\rho=a m \sin \theta$ :

$S_{\omega}=\frac{2 q^{2}}{\pi c} \frac{k^{2}}{\beta^{2}} \int_{0}^{\theta_{m}} \mathrm{~d} \theta \sin \theta \cos \theta \Phi^{2}(r, \alpha, k, k \sin \theta)$,

where $\theta_{m}=\arcsin (d / a m)$ is the angular acceptance of the detector and

$$
\begin{array}{r}
\Phi(r, \alpha, k, \delta)=\frac{\delta}{\alpha^{2}+\delta^{2}}-\frac{\alpha r}{\alpha^{2}+\delta^{2}}\left[\delta K_{1}(\alpha r) J_{0}(\delta r)\right. \\
\left.+\alpha J_{1}(\delta r) K_{0}(\alpha r)\right]-\int_{0}^{r} \mathrm{~d} \rho_{s} J_{0}\left(k \rho_{s}\right) J_{1}\left(\delta \rho_{s}\right) .
\end{array}
$$

Here $K_{n}$ is the modified Bessel function of the $\mathrm{n}$-th order.

Figures 2 and 3 show the spectra calculated by using Eq. (6) for parameters and frequency ranges typical for bunch length measurements, and normalized to the corresponding spectra from an infinite screen.

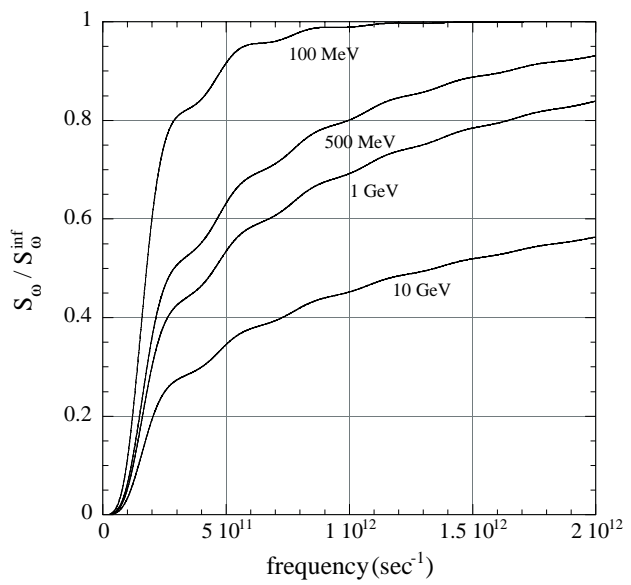

Figure 2: Spectra $S_{\omega}$ of TR in the first scheme of measurements, for a screen with radius of $20 \mathrm{~mm}$ and a detector angular acceptance of $0.05 \mathrm{rad}$.

\subsection{Spectrum of TR from a screen in the focal plane of a lens}

The second scheme under consideration (Fig. 1b) is a simplified geometry normally used in autocorrelation interferometric measurements, when the screen is placed in the front focal plane of a converging lens (parabolic mirror) to produce, behind the lens, a quasi-parallel photon beam.

The analysis performed for a thin lens with diameter $2 D$ and focal length $f$ results in the expression for the field identical to Eq. (2) and (4) if one puts $a=f$ and $m=1$. The power spectrum, in the infinite lens approximation, is, therefore, given by Eq. (6) with

$$
\theta_{m}=\arcsin (d / f) \text {. }
$$




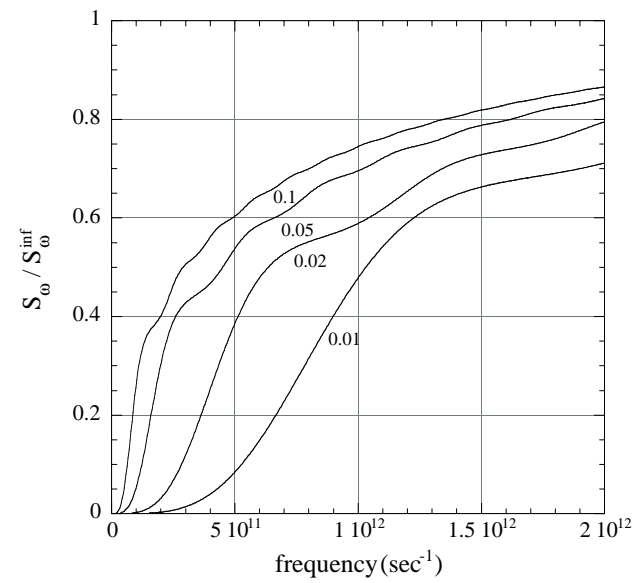

Figure 3: Spectra of TR $S_{\omega}$ in the first scheme of measurements for a screen radius of $20 \mathrm{~mm}$ and beam energy of $1 \mathrm{GeV}$. Numbers next to the curves are detector angular acceptances in radians.

The similarity between the first and second schemes can be seen by simple ray tracing: the effect of the lens is basically to "draw" the detector towards the screen, resulting in an increase of the angular acceptance of the system.

\subsection{Spectrum of TR in the screen's image plane}

Here the diaphragm of the first scheme is replaced by a lens of the same size and $a$ and $b$ are chosen such that the condition $1 / a+1 / b=1 / f$ is satisfied ( Fig. 1c). In this geometry the screen is simply imaged onto the detector. The expression for the field is given by

$$
\begin{aligned}
E_{x, y}(P, \omega)= & \frac{q}{2 \pi^{2} v} \frac{k^{2}}{a b} \int \mathrm{d} \rho_{s} \rho_{s} \int \mathrm{d} k_{\perp} \frac{k_{\perp}^{2} J_{1}\left(k_{\perp} \rho_{s}\right)}{k_{\perp}^{2}+\alpha^{2}} \\
& \times \mathrm{e}^{\mathrm{i}(k / 2 a) \rho_{s}^{2}} \int \mathrm{d} \varphi\left\{\begin{array}{l}
\cos \varphi \\
\sin \varphi
\end{array}\right\} \mathcal{L}(p),
\end{aligned}
$$

where, as before, $\mathcal{L}$ is the pattern resulting from the diffraction on the lens, that in this case is given by the well-known expression

$$
\begin{gathered}
\mathcal{L}(p)=\frac{2 \pi a D}{k p} J_{1}\left(\frac{k D}{a} p\right), \\
p=\sqrt{\varrho^{2}+\rho_{s}^{2}+2 \varrho \rho_{s} \cos (\varphi-\chi)},
\end{gathered}
$$

with $\varrho=\rho / M$, where $M=b / a$ is the lens magnification.

For the infinite lens and neglecting the phase factor, the total power spectrum can be given in the form

$$
S_{\omega}=\frac{2 q^{2}}{\pi c} \frac{1}{\beta^{2}} \int_{0}^{d / M} \mathrm{~d} \varrho \varrho\left[\alpha K_{1}(\alpha \varrho)-\frac{J_{0}(k \varrho)}{\varrho}\right]^{2} .
$$

In Eq. (11) we imply that $d / M \leq r$, while generally one should integrate from 0 to $\min \{d / M, r\}$.

We want to attract attention to the identical role of the screen and detector dimensions in affecting the spectrum. In fact, since the intensity distribution in the image plane is just a "magnified" image of that in the source plane, both screen and detector are equivalent in producing restrictions on the transverse region over which the power spectrum must be calculated; namely, the spectrum is only determined by the minimum values of $d / M$ and $r$.

\section{EFFECT OF THE SCREEN SIZE IN CTR BASED BUNCH LENGTH MEASUREMENTS}

Considering a low-frequency distortion of the TR spectrum due to a finite-size screen in the context of bunch length measurements one can expect that its effect on the accuracy of the bunch information retrieval may be sufficiently small as long as the corrupted portion of the spectrum is negligible compared to the frequency content of the bunch structure represented by the bunch form-factor. For the gaussian-shaped beam the following qualitative criterion can be used to estimate whether for a given bunch length $\sigma_{z}$ the effect of the screen size is important

$$
\sigma_{z}<c / \sqrt{2} \omega_{\mathrm{ch}}
$$

Here $\omega_{\mathrm{c} h}$ is a characteristic cutting frequency of the spectrum due to the effect. If, as usual for such kind of problems, one defines the cutting frequency as a $10 \%$ dropoff of spectra from the high-frequency plateau, simple approximate relations for $\omega_{\mathrm{ch}}$ may be obtained by analyzing Eqs. (11) and (6), respectively

$$
\omega_{\mathrm{ch}}\left(\mathrm{sec}^{-1}\right)=3.3 \cdot 10^{9} E^{0.87}(\mathrm{MeV}) / \sqrt[4]{\theta_{m}(\mathrm{r} a d)}
$$

and

$$
\omega_{\mathrm{ch}}\left(\mathrm{sec}^{-1}\right)=8.3 \cdot 10^{10} E^{0.87}(\mathrm{MeV}) / d(\mathrm{~mm}) .
$$

\section{REFERENCES}

[1] N.F.Shul'ga and S.N.Dobrovol'skii, Pis'ma Zh. Eksp. Teor. Fiz. 65 (1997) 581 [JETP Lett. 65 (1997) 611]. N.F.Shul'ga, S.N.Dobrovol'skii and V.G.Syshchenko, Nucl.Instr. and Meth. in Phys. Res. B 145 (1998) 180 .

[2] A.P.Potylitsin, Nucl. Instr. and Meth. in Phys. Res. B 145 (1998) 169.

[3] W.Barry, AIP Conference Proceedings 390 (1997) 173; Proc. of the 7th Beam Instrumentation Workshop, Argonne, May 1996.

[4] A.Murokh, J.B.Rosenzweig, M.Hogan, H.Suk, G.Travich and U.Happek, Nucl. Instr. and Meth. in Phys. Res. A 410 (1998) 452 .

[5] M.Castellano and V.A Verzilov, Phys. Rev. ST- Accel. Beams 1 (1998) 062801.

[6] M.Born and E.Wolf, Principles of Optics (Pergamon Press, New York, 1965). 